\title{
Childhood type 2 diabetes: Risks and complications (Review)
}

\author{
YING XUE, MIN GAO and YIQING GAO \\ Department of Endocrinology, Xuzhou Children's Hospital, Xuzhou, Jiangsu 221002, P.R. China
}

Received November 27, 2015; Accepted March 3, 2016

DOI: $10.3892 /$ etm.2016.3654

\begin{abstract}
The universal endocrine pathological state affecting young individuals and adults is type 2 diabetes mellitus, which has seen a significant increase in the last 30 years, particularly in children. Genetic and evnironmental factors are the causative agents for this pathological state in children. This rapid and wide spread of the disease can be controlled by enforcing amendments in environmental factors such as diet, physical activities and obesity. In young infants breastfeeding may be a key modulator of the disease. Associated disorders co-observed in the patients of type 2 diabetes mellitus include renal failure, heart problems and circulatory dysfunctionalities, such as cardiac failure and vision disability. These associated disorders become more pronounced in young patients when they reach puberty. To overcome the lethal outcomes of the disease, early screening of the disease is crucial. The present review focused on the latest updates in the field, as well as plausible risks and complications of this pathological state.
\end{abstract}

\section{Contents}

1. Introduction

2. Childhood DMII prevalence

3. Childhood DMII etiology

4. Genetic non-modifiable risk factors

5. Modifiable risk factors

6. Important effects of childhood DMII

7. Vision impairment

8. Conclusions

\section{Introduction}

Diabetes is considered a curable disease, however, associated diseases make the condition complex (1). In simple terms, diabetes is a pathological state that does not allow an affected

Correspondence to: Dr Min Gao, Department of Endocrinology, Xuzhou Children's Hospital, 18 Sudibei Road, Xuzhou, Jiangsu 221002, P.R. China

E-mail: g696441@163.com

Key words: childhood diabetes, risks, complications, diabetes type 2 individual to properly metabolize glucose intake. Diabetes mellitus is classified as type 1 (DMI) and type 2 (DMII). However, it is difficult to identify each type separately (2). DMII is associated more with highly complex co-pathological states and is predicted in advance by various risk factors.

Normal levels of glucose in a healthy individual vary from 70 to $125 \mathrm{mg} / \mathrm{dl}$. However, observance of exceptionally high levels of glucose in the blood, known as hyperglycemia, is a certain indicator of diabetes. The body resistance to insulin and improper insulin release due to abnormality in $\beta$ cells of pancreas are the root causes of hyperglycemia (3). Thus, the patients affected by hyperglycemia in DMI, due to non-availability of insulin, become totally dependent on external insulin supplements. Furthermore, although some DMI patients may produce normal insulin, the body resistance to insulin makes this insulin ineffective in glucose management (4), a condition medically termed as insulin resistance.

Three causative processes are responsible for insulin resistance in DMII. The first and foremost reason is defect in the normal signalling circuit of the physiological system leading to incorrect timing of the release of insulin by the pancreas, ultimately resulting in abnormally high glucose levels. The second reason for insulin resistance is a faulty glucose transporter (1). The third reason for this pathological state is the accumulation of lipid metabolites in body tissues such as liver and arteries causing lipotoxicity, which in turn results in insulin intolerance. Lipotoxicity is also the result of excess tissue fat causing insulin resistance indirectly (5). The treatment plan for DMII is dependent on the causative reason responbile for the pathological state and is different for the reasons identified behind insulin resistance.

\section{Childhood DMII prevalence}

DMII was originally considered an adult disease, but was verified as a prominent childhood disease in the 1980s. The Center for Disease Control and Prevention estimates that in the coming 40 years the rate of childhood DMII is likely to rapidly rise and its spread is expected to be 4-fold that of the current situation (6). Currently, there is a rapid rise in the confirmed cases of DMII in young patients aged 4-5 years (7). Thus, the problem is magnified in child patients as compared to adults.

\section{Childhood DMII etiology}

The factors responsible for DMII are genetic as well as environmental, making the etiology of DMII highly 
heterogeneous (8). Factors that cannot be modified or mitigated are genetic risk factors as they are irreversible in nature and are present from birth. On the other hand, modifiable factors are environmental and include diet and lifestyle. These environmental factors may be altered to prevent the onset as well as development of DMII.

\section{Genetic non-modifiable risk factors}

General. The three major non-modifiable risk factors responsible for DMII include intrauterine exposure, family history, and ethnicity (9). These factors contribute towards the development of this lethal disease in their respective unique manner.

Intrauterine exposure. The most prominent factor of genetic nature responsible for childhood DMII is intrauterine exposure to maternal diabetes (10). However, the diabetes caused in the young infant is DMI, DMII, or gestational diabetes. Medically it has been proven that maternal hyperglycemia and unstable balance between insulin and glucose are the core factors responsible for DMII in young infants (11). Despite advancements in the medical management of blood glucose levels of the mother during pregnancy, it has been observed that intrauterine exposure to maternal diabetes is lethal for infants. The resultant pathologies observed in children who have developed DMII are hyperglycemia, macrosomia, together with the association of improper development of the endocrine system (11).

Hyperglycemia. The state of hyperglycemia in the pregnant mother with diabetes leads to continuous exposure of high levels of glucose to the developing fetus throughout the pregnancy (11). The route of increased glucose transfer to the fetus occurs via the umbilical cord, which in turn stimulates the developing pancreas to elevate insulin secretion in order to prevent hyperglycemia. Thus, the infants that are born under these circumstances are usually adapted to high glucose levels $(12,13)$. Therefore, the unavailability of high glucose levels following birth results in decreased insulin production in newborn infants and these marked changes in the environment and glucose intake cause premature insulin resistance in childhood, which essentially constitutes a growth point for the development of DMII (14). In addition, the above abnormal conditions directly affect the young endocrine control in the infant, leading to associated pathological states in the near future as discussed earlier in this study. The severity as well as duration of the hyperglycemic conditions of the mother determine the extent of DMII disease in the newborn infant.

Macrosomia. The condition of abnormally high birth weight in neonates is medically known as macrosomia. The excessive weight is usually the result of the mother's defective endocrine system or the effects of existing metabolic syndrome. Diabetic mothers who undergo treatment and whose glucose levels are well-controlled by medical personnel may give birth to a child with overweight or macrosomic infant (15). The causative factor responsible for the conditon of macrosomia is the rapid and continuous formation of fat tissue in the infant despite regulated glucose levels in the mother. Previous findings confirmed that an infant's adipose growth is directly affected by intrauterine metabolic factors that cause malfunctioning of the infant endocrinal system (16).

Endocrine system complications. As mentioned above, exposure to abnormally high glucose levels in the mother result in impaired endocrine development in neonates. The important molecular step involved in disturbing the normal physiological system of a young infant is the excess production of insulin by the infant endocrine system, leading to the state of hyperinsulinism (17). The initiation of hyperinsulinism in the first trimester greatly affects the neural development of the infant as this period is crucial for brain development. Hyperinsulinism at this stage direclty results in a faulty hypothalamic center that under normal circumstances regulates endocrine function. This results in significant growth maladaptation in the growing infant, which leads to childhood DMII (18). The above associated complications of the endocrine system along with DMII condition in these young infants makes them a high-risk population of individuals with strong possiblities of having obesity and other associated diseases in their adult life (19).

Family history and DMII. Family history of the disease is a crucial factor responsible for the spread of DMII among neonates. Children with an immediate family member that has been diagnosed with this pathological state are at high risk for the development of childhood DMII as the genetic makeup of the infant promotes the feasibility of this lethal disease $(20,21)$. In mathematical terms, the approximate percentage of risk of development of DMII is $40 \%$ when one of the parents is affected with diabetes. On the other hand, when both the parents have diabetes, the likelihood of development of DMII in neonates increases to $70 \%$ (22). The genetic makeup of affected parents basically promotes obesity in the young patient, which in turn is useful in the diagnosis of DMII. A previous study suggested the possibility of a confirmed genetic factor, such as a gene or transcription factor, responsible for control of glucose intake and insulin regulation (23). Previous findings indicated an association of TCF7L2 and MTNR1B genes with the development of DMII (24). Thus, it is crucial for children of affected parents to get screened early in order that proper treatment planning can be undertaken in a timely manner. Early diagnosis of DMII is one of the major research hotspots and the stress is on the identification of highly specific genetic markers for the diagnosis of DMII in young infants.

\section{Modifiable risk factors}

General. Irreversible factors are responsible for DMII. Reversible factors are also crucial in the spread of diabetes. These factors can be controlled by human, contributing to the well-being of diabetic patients. The lifestyle of an individual is the self-contained factor that can be modified by the individuals themselves (25). Other factors in children for which parents are responsible include diet and breast feeding.

Breastfeeding in infancy. The proper health of a child is the result of proper breastfeeding in his/her infancy (26). Improper breastfeeding is a prime factor responsible for the development 
of obesity and metabolic syndrome in future (27). Studies have identified a $24 \%$ decrease in the risk of prevalence of childhood DMII and associated obesity in children with access to proper breastfeeding (28). Furthermore, a systemic review of 17 studies has revealed a direct correlation between duration of breastfeeding and risk of development of childhood DMII and obesity in neonates (29). One of these 17 studies (29) emphasized that children in the 9-14-year age group who were breastfed for longer durations were not affected by obesity or any other metabolic syndrome disease, including childhood DMII (29). Thus, breastfeeding is an important aspect in the prevention of this chronic disorder.

Obesity and dietary habits. Obesity is the second major reversible factor responsible for this pathological state. Weight is the deciding criterion for distinguishing high- and low-risk children of developing DMII. Children of a healthy weight are less likely to be affected by childhood DMII as opposed to obese children who are at high risk. There is also a direct interdependence of obesity with childhood DMII (30). The important linking factor between obesity and DMII is insulin resistance, which results in the excess of free fatty acids (FFAs) in obese infants (31). These excess of FFAs in obese infants contribute towards lipolytic activity, an important causative factor for DMII, as discussed in earlier sections. On the other hand, decline in physical activity also promotes insulin resistance (32). By increasing physical activity the growth of muscle volume can be stimulated, resulting in enhanced glucose transport and a decrease in insulin demand. In this manner, enhanced physical activity promotes the efficient utilization of glucose intake and maintains a check over the development of DMII (33). Modification of diet risk of development of DMII is also greatly reduced. Previous findings have shown that excessive intake of carbohydrates in diet favoured the development of childhood DMII (34).

\section{Important effects of childhood DMII}

General. There are significant long-term efffects of childhood DMII, such as life-threatening pathologies including peripheral neuropathy $(35,36)$. Cardiac complications, renal failure, and vision impairment are other well-reported complications of DMII.

Cardiac complications. Common complications associated with DMII are cardiac ones. Important cardiac alterations in the literature included cardiomyocyte-altered metabolism, changes in cardiac insulin signaling, general calcium control and the stimulation of vascular as well as cardiac fibrosis (37). Fibrotic build up along with overworking of cardiac muscles is also common among DMII patients (38). These complications lead to other well-known complications of cardiac pathology including hyperlipidemia, coronary artery disease, hypertension and diabetic cardiomyopathy $(6,39)$. Previous findings suggested that $50 \%$ of infants confirmed with DMII usually develop cardiac complications such as contractility dysfunction and cardiac autonomic neuropathy (40).

Complications of renal system. The renal complications associated with DMII are also frequently recorded and are mainly the result of poor flow of blood towards kidneys, a condition medically termed chronic kidney disease (41). The condition of hyperglycemia results in damage of masangial cells that ultimately blocks kidney function. This function blockage of the kidneys leads to albuminuria (42).

\section{Vision impairment}

Vision impairment in DMII patients is mainly due to diabetic retinopathy. The high blood glucose condition observed in DMII patients results in microvascular damage to the retina that ultimately impacts on the vision of affected patients (43). Biochemical factors such as sorbitol accumulation, oxidative stress resulting in tissue damage, protein kinase $\mathrm{C}$ activation, and dysfunction of the renin-angiotensin-aldosterone system collectively contribute towards vision impairment. The retinal vessels are also damaged by inflammation. Thus, early diagnosis of DMII in children is imperative to prevent adverse complications of vision impairment.

\section{Conclusions}

The present review has demonstrated that the number of children diagnosed with DMII is on the increase, thereby elevating the spread of serious complications associated with the disease. Focused research is essential for the efficient screening and understanding of the disease to reduce the risks and complications associated with childhood DMII.

\section{References}

1. Taylor R: Type 2 diabetes: etiology and reversibility. Diabetes Care 36: 1047-1055, 2013.

2. Rao PV: Type 2 diabetes in children: clinical aspects and risk factors. Indian J Endocrinol Metab 19 (Suppl 1): S47-S50, 2015.

3. Ozougwu JC, Obimba KC, Belonwu CD and Unakalamba CB: The pathogenesis and pathophysiology of type 1 and type 2 diabetes mellitus. J Physiol Pathophysiology 4: 46-57, 2013.

4. Copeland KC, Silverstein J, Moore KR, Prazar GE, Raymer T, Shiffman RN, Springer SC, Thaker VV, Anderson M, Spann SJ, et al; American Academy of Pediatrics: Management of newly diagnosed type 2 Diabetes Mellitus (T2DM) in children and adolescents. Pediatrics 131: 364-382, 2013.

5. DeFronzo RA: Insulin resistance, lipotoxicity, type 2 diabetes and atherosclerosis: the missing links. The Claude Bernard Lecture 2009. Diabetologia 53: 1270-1287, 2010.

6. Vinicor F and Jack LJ: 25 years and counting: Centers for Disease Control and Prevention identifies opportunities and challenges for diabetes prevention and control. Ann Internmed 140: 943-944, 2004.

7. Hannon TS, Rao G and Arslanian SA: Childhood obesity and type 2 diabetes mellitus. Pediatrics 116: 473-480, 2005.

8. No authors listed: Type 2 diabetes in children and adolescents-A reality in pediatric pathology. Romanian J Pediatr 59: 278-283, 2010 .

9. Kommoju UJ and Reddy BM: Genetic etiology of type 2 diabetes mellitus: A review. Int J Diabetes Dev Ctries 31: 51-64, 2011

10. McIntyre HD, Thomae MK, Wong SF, Idris N and Callaway LK: Pregnancy in type 2 diabetes mellitus - problems and promises. Curr Diabetes Rev 5: 190-200, 2009.

11. Lin RS, Lee WC, Lee YT, Chou P and Fu CC: Maternal role in type 2 diabetes mellitus: Indirect evidence for a mitochondrial inheritance. Int J Epidemiol 23: 886-890, 1994.

12. Ma RC, Tutino GE, Lillycrop KA, Hanson MA and Tam WH; Maternal diabetes, gestational diabetes and the role of epigenetics in their long term effects on offspring. Prog Biophys Mol Biol 118: 55-68, 2015.

13. Donovan LE and Cundy T: Does exposure to hyperglycaemia in utero increase the risk of obesity and diabetes in the offspring? A critical reappraisal. Diabet Med 32: 295-304, 2015. 
14. Ruchat SM, Hivert MF and Bouchard L: Epigenetic programming of obesity and diabetes by in utero exposure to gestational diabetes mellitus. Nutr Rev 71 (Suppl 1): S88-S94, 2013.

15. Small M, Cameron A, Lunan CB and MacCuish AC: Macrosomia in pregnancy complicated by insulin-dependent diabetes mellitus. Diabetes Care 10: 594-599, 1987.

16. Catalano PM and Hauguel-De Mouzon S: Is it time to revisit the Pedersen hypothesis in the face of the obesity epidemic? Am J Obstet Gynecol 204: 479-487, 2011.

17. Stanley CA: Advances in diagnosis and treatment ofhyperinsulinism in infants and children. J Clin Endocrinol Metab 87: 4857-4859, 2002.

18. Nielsen JH, Haase TN, Jaksch C, Nalla A, Søstrup B, Nalla AA, Larsen L, Rasmussen M, Dalgaard LT, Gaarn LW, et al: Impact of fetal and neonatal environment on beta cell function and development of diabetes. Acta Obstet Gynecol Scand 93: 1109-1122, 2014.

19. Dorajoo R, Liu J and Boehm BO: Genetics of Type 2 Diabetes and Clinical Utility. Genes (Basel) 6: 372-384, 2015.

20. Mohlke KL and Boehnke M: Recent advances in understanding the genetic architecture of type 2 diabetes. Hum Mol Genet 24 (R1): R85-R92, 2015.

21. Mendelson M, Cloutier J, Spence L, Sellers E, Taback S and Dean H: Obesity and type 2 diabetes mellitus in a birth cohort of First Nation children born to mothers with pediatric-onset type 2 diabetes. Pediatr Diabetes 12: 219-228, 2011.

22. Ahlqvist E, Ahluwalia TS and Groop L: Genetics of type 2 diabetes. Clin Chem 57: 241-254, 2011.

23. Santoro N, Amato A, Grandone A, Brienza C, Savarese P, Tartaglione N, Marzuillo P, Perrone L and Miraglia Del Giudice E: Predicting metabolic syndrome in obese children and adolescents: Look, measure and ask. Obes Facts 6: 48-56, 2013.

24. Gunderson EP: Breast-feeding and diabetes: Long-term impact on mothers and their infants. Curr Diab Rep 8: 279-286, 2008.

25. McGavock J, Dart A and Wicklow B: Lifestyle therapy for the treatment of youth with type 2 diabetes. Curr Diab Rep 15: 568 , 2015.

26. Pereira PF, Alfenas RC and Araujo RM: Doesbreastfeeding influence the risk of developing diabetes mellitus in children? A review of current evidence. J Pediatr (Rio J) 90: 7-15, 2014.

27. Smithers LG, Kramer MS and Lynch JW: Effects of Breastfeeding on Obesity and Intelligence: Causal Insights From Different Study Designs. JAMA Pediatr 169: 707-708, 2015.

28. Bartz S and Freemark M: Pathogenesis and prevention of type 2 diabetes: Parental determinants, breastfeeding, and early childhood nutrition. Curr Diab Rep 12: 82-87, 2012.

29. Rabbitt A and Coyne I: Childhood obesity: Nurses' role in addressing the epidemic. Br J Nurs 21: 731-735, 2012.
30. Yaturu S: Obesity and type 2 diabetes. J Diabetes Mellitus 1: 79-95, 2011.

31. Tompkins C, Soros A, Sothern M and Vargas A: Effects of physical activity on diabetes management and lowering risk for type 2 diabetes. Am J Health Educ 40: 286-290, 2009.

32. Lumb A: Diabetes and exercise. Clin Med Lond 14: 673-676, 2014.

33. Donin AS, Nightingale CM, Owen CG, Rudnicka AR, Jebb SA, Ambrosini GL, Stephen AM, Cook DG and Whincup PH: Dietary energy intake is associated with type 2 diabetes risk markers in children. Diabetes Care 37: 116-123, 2014.

34. Gardner JD, Murray DB and Wold LE: Cardiac dysfunction in diabetes. Life Sci 92: 599-600, 2013.

35. Constantino MI, Molyneaux L, Limacher-Gisler F, Al-Saeed A, Luo C, Wu T, Twigg SM, Yue DK and Wong J: Long-term complications and mortality in young-onset diabetes: Type 2 diabetes is more hazardous and lethal than type 1 diabetes. Diabetes Care 36: 3863-3869, 2013.

36. Song SH: Complication characteristics between young-onset type 2 versus type 1 diabetes in a UK population. BMJ Open Diabetes Res Care 3: e000044, 2015.

37. Mandavia CH, Aroor AR, Demarco VG and Sowers JR: Molecular and metabolic mechanisms of cardiac dysfunction in diabetes. Life Sci 92: 601-608, 2013.

38. Cheung BM and Li C: Diabetes and hypertension: is there a common metabolic pathway? Curr Atheroscler Rep 14: 160-166, 2012.

39. Jørgenrud B, Jalanko M, Heliö T, Jääskeläinen $P$, Laine $M$, Hilvo M, Nieminen MS, Laakso M, Hyötyläinen T, Orešič M, et al: The Metabolome in Finnish Carriers of the MYBPC3-Q1061X Mutation for Hypertrophic Cardiomyopathy. PLoS One 10: e0134184, 2015.

40. Schnell O, Hammer K, Muhr-Becker D, Ziegler A, Weiss M, Tatsch $\mathrm{K}$ and Standl E: Cardiac sympathetic dysinnervation in Type 2 diabetes mellitus with and without ECG-based cardiac autonomic neuropathy. J Diabetes Complications 16: 220-222, 2002.

41. Min TZ, Stephens MW, Kumar P and Chudleigh RA: Renal complications of diabetes. Br Med Bull 104: 113-127, 2012.

42. Carroll EW, Scott JA and Curtis R (eds): Disorders of visual function. In: Pathophysiology: Concepts of Altered Health States. Wolters Kluwer Health/Lippincott Williams \& Wilkins, Philadelphia, PA, pp1388-1426, 2009.

43. Cheung N, Mitchell P and Wong TY: Diabetic retinopathy. Lancet 376: 124-136, 2010. 